\title{
Thermal Conversion of Waste Plastics (HDPE, PP and PS) to Produce Mixture of Hydrocarbons
}

\author{
Moinuddin Sarker*, Mohammad Mamunor Rashid, Mohammed Molla, Muhammad Sadikur Rahman
}

Department of Research and Development, Natural State Research, Inc., 37 Brown House Road (2nd Floor), Stamford, CT-06902, USA

\begin{abstract}
Thermal conversion process was applied with three types of waste plastic mixture and waste plastics mixture to liquid hydrocarbon fuel production in present of oxygen under laboratory Labconco fume hood without adding any kind of catalyst. Utilization of the process described can reduce the impact of waste plastics significantly. Thermal decomposition of the most common plastics such as high density polyethylene (HDPE), polypropylene (PP) and polystyrenes (PS) has been conducted to produces a mixture of hydrocarbons. The thermal process applied of mixed waste plastics using a steel reactor at $25-430^{\circ} \mathrm{C}$ has been investigated. The reactor was connected with standard condensation unit with water circulator system. Waste plastics are broken down into shorter chain hydrocarbon compounds from long chains during the thermal conversion process without adding catalyst or chemical. Produced fuel was analysis by using GC/MS, FT-IR and DSC and from GC/MS analysis result showed hydrocarbon chain range $\mathrm{C}_{3}$ to $\mathrm{C}_{28}$ into fuel and produce fuel can be use as feed stock refinery or power plant for electricity generation or can use for internal combustion engine.
\end{abstract}

Keywords Waste Plastic, Thermal Decomposition, Hydrocarbon, Condensation, Polyethylene, Polystyrene, polypropylene

\section{Introduction}

The present rate of economic growth is unsustainable without saving of natural energy like crude oil, gas or coal. Thus mankind will soon have to rely on alternate/renewable energy sources like biomass, hydropower, geothermal energy, wind energy, solar energy, and nuclear energy etc. On the other hand, suitable waste management strategy is another important aspect of sustainable development. Plastics have been one of the materials with the fastest growth because of their wide range of applications due to versatility and relatively low cost. Since the duration of life of plastic products is relatively small, there is a vast plastic waste stream that reaches each year to the final recipients creating a serious environmental problem. Again, because disposal of post consumer plastics is increasingly being constrained by legislation and escalating cost, there is considerable demand for alternatives to disposal or land filling.

Advanced research in the field of green chemistry could yield biodegradable/green polymers but is too limited at this point of time to substitute the non-biodegradable plastics in different applications. Many research projects have been undertaken on chemical recycling of waste plastics to fuel and monomer. Some examples of these achievements

\footnotetext{
* Corresponding author:

msarker@naturalstateresearch.com (Moinuddin Sarker)

Published online at http://journal.sapub.org/ajee

Copyright (C) 2012 Scientific \& Academic Publishing. All Rights Reserved
}

include pilot, demonstration and commercial plants processing various types of waste plastic in Germany, Japan, USA and India and elsewhere.

The rapid rate of plastic consumption throughout the world has led to the creation of increasing amounts of waste plastic and this in turn poses greater difficulties for disposal. This is due to the fact that duration of life of plastic wastes is very small[1] and depending on the area of application, the service life of plastic product ranges from 1 to 35 years[2]. The weighted average service life of all plastics products is different in different countries based on the counties life style and economy. Plastic wastes can be classified as industrial and municipal waste plastic according to their origins; these groups have different qualities and properties and are subjected to different management strategies[3]. Waste plastics represent a large part of municipal wastes; furthermore huge amount of waste plastic arise as a by product or faulty product in industry and agriculture[4]. Thermo plastics are composed of polyolefin such as high density polyethylene (HDPE), low density polyethylene (LDPE), polypropylene (PP), polystyrene (PS), polyethylene terephthalate (PETE) and polyvinyl chloride (PVC)[5] and can be recycled. On the other hand thermosets mainly include epoxy resins and polyurethanes and these cannot be recycled.

Due to population increase, the demand for plastic products has steadily increased over the last 40 years. Since plastics are non-biodegradable, they cannot be easily returned to the natural carbon cycle; hence the life cycle of 
plastic materials ends at waste disposal facilities[6]. There are several methods for disposal of municipal and industrial plastic waste; i.e. landfill, incineration (energy recovery), true material recycling (similar recycled product or monomer recovery. The largest amount of waste plastic is disposed of by land filling $(65-70 \%)$ and incineration (20-25\%). Recycling is only about $10 \%$. Moreover, the problem of waste cannot be solved by land filling and incineration, because suitable and safe deposits are expensive, and incineration stimulates the growing emission of harmful, greenhouse gases. Thermal recycling of waste polymers under different catalytic and thermal circumstances has been well investigated by researchers[7-11]. Other important parameters for waste polymer degradation are residence time, and the chemical structure of polymer. The effects of these parameters have been less investigated and described. The packaging industry and other human activities use a huge amount of PE and PP. Usually there are multiple municipal companies that handle the collecting and sorting of these waste plastics, but due to the vast amount and large diversion the sorting do not always consist of specific plastic type, and at the end we end up with a mixed waste plastic types.

The subject of this paper is demonstrate the process of converting the vast amount of waste plastic to useful hydrocarbon fuel utilizing thermal degradation process. The process was carried out utilizing a steel reactor using a temperature range of 25 to $430^{\circ} \mathrm{C}$. The final product was analysed and the results are presented in the paper.

\section{Materials and Methods}

\subsection{Materials}

The raw materials used for the experiment were HDPE, PP and PS. Raw samples were collected form Stamford municipality. The collected waste plastics were dirty and come with foreign materials. HDPE waste plastic sample was white color milk container, PP waste plastic samples was white color food container and PS waste plastic samples was transparent food containers. All foreign materials were separated by manually then collected waste plastic were cleaned and wash with liquid (7th generation company) soap. Washed waste plastics were cut into small pieces and grinded into 3-4 $\mathrm{mm}$ size. The proportion of the waste plastic sample used in the experiment is shown in table 1. The raw materials elemental composition is presented in table 2 . Table 2 elemental analysis preformed by ASTM test method ASTM D5291_a. From analysis result we noticed that $\mathrm{C}, \mathrm{H}$ and $\mathrm{N} \%$ of the sample is less than $100 \%$ because these waste plastics have other impurities. During the plastic manufacturing process different types of additives (3-5\%) are added to obtain a desired shape, hardness and appearance, and due to these additives we believe we have a shorter percentage of $\mathrm{C}, \mathrm{H}$ and $\mathrm{N}$.

\subsection{Experiment}

The washed out waste plastics are cut into small pieces to fir the reactor. Prior to the reactor feeding process the plastics samples was analysed using Thermogravimetric (TGA, Payris-1) analyser for onset temperature measurement, FT-IR (Spectrum 100) for functional group tracing and functional group bend energy determination, Elemental analyser (EA-2400) for carbon, hydrogen and nitrogen percentage analysis and finally Gas Chromatography and Mass Spectrometer (GC/MS) with pyroprobe for raw waste plastics compound structure determination. The mixture of waste plastics sample was place in the reactor chamber for liquefaction process with a temperature range of $25-430^{\circ} \mathrm{C}$. A total of $700 \mathrm{gm}$ of solid was plastics were used for the reactor process. Then reactor was covered and screw was tighten at the end condenser unit. One end of a condenser unit was hooked with the reactor and another end was hooked up with a collection container. For fuel condensation purposed water temperature was $20^{\circ} \mathrm{C}$. Collection unit top side was hook up light gas cleaning device and collection tank bottom part was hook up RCI fuel purification device for fuel purification at the end light gas storage system was hook up with light gas cleaning device with small pump. $25^{\circ} \mathrm{C}$ to $430^{\circ} \mathrm{C}$ temperature was used for the liquefaction process and process was fully closed system but without vacuum (see figure 1). There were no catalysts involved in the conversion process. The starting temperature setup was $25^{\circ} \mathrm{C}$ and then $25^{\circ} \mathrm{C}$ to increase temperature $200{ }^{\circ} \mathrm{C}$ quickly for quick melting of the mixture sample. In this experiment we noticed that when the temperature was increased quickly from room temperature to $200^{\circ} \mathrm{C}$ some vapor started to exhaust out of the condenser unit. Again when temperature is gradually increased from $200^{\circ} \mathrm{C}$ to $280^{\circ} \mathrm{C}$ we received liquid hydrocarbon fuel. This specific temperature range was used because the melting point of the HDPE is $130{ }^{\circ} \mathrm{C}$, PP melting point is $160^{\circ} \mathrm{C}$ and Polystyrene's melting point is $240^{\circ} \mathrm{C}$. The experiment temperature was increased every 15 minute by $10^{\circ} \mathrm{C}$ and until the max of $430^{\circ} \mathrm{C}$. During the conversion process we notice that the entire vapours were not condensed and some portion of vapours came out as a light gas. Light gas was mixture with methane, ethane, propane and butane. An alkali filled gas cleaning system was used to decontaminate and purify the gas. The alkali solution used in the cleaning system was $0.25(\mathrm{~N})$ sodium bicarbonate solution. The light gas is collected into a Teflon bag using suction system for future usages or testing purpose. The liquid fuel was purified using a RCI purification unit and this unit has centrifugal force and micro filter technology which removes fuel sediment and water portion. We believe that the water portion may arise the atmospheric moisture. The liquid fuel yield percentage was $90 \%$, light gas was $4.67 \%$ and solid black residue is $5.33 \%$. 
Table 1. Composition of the waste plastics mixture for experiment

\begin{tabular}{cc}
\hline Materials & Wt.\% \\
\hline HDPE & 40 \\
PP & 30 \\
PS & 30 \\
\hline
\end{tabular}

Table 2. Waste plastics elemental composition (Wt. \%) by EA-2400

\begin{tabular}{cccc}
\hline Sample Name & Carbon $\%$ & Hydrogen $\%$ & Nitrogen \% \\
\hline HDPE & 83.57 & 14.78 & $<0.30$ \\
PP & 79.93 & 14.17 & $<0.30$ \\
PS & 78.60 & 7.21 & $<0.30$ \\
\hline
\end{tabular}

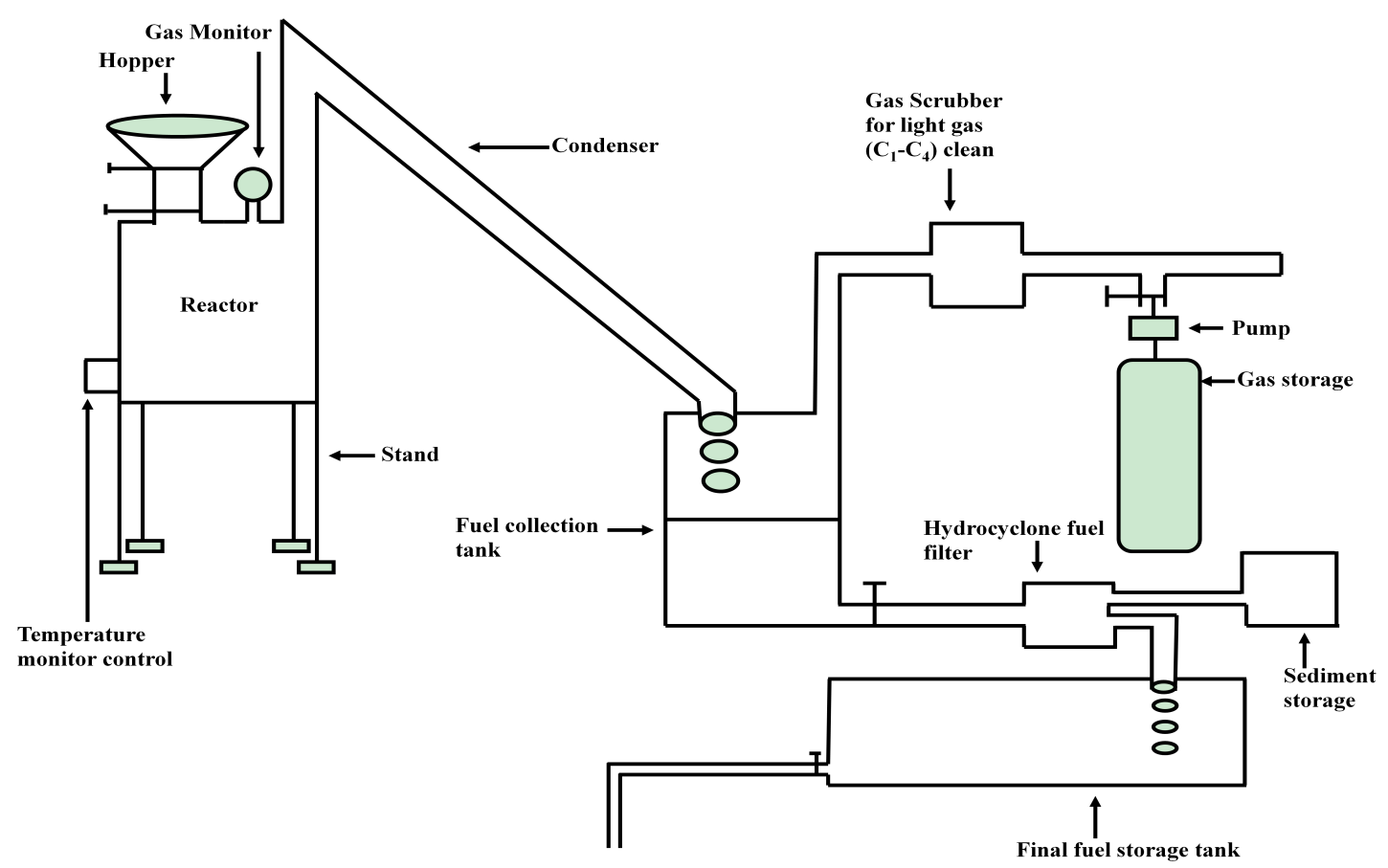

Figure 1. Diagram of HDPE, PP and PS waste plastics mixture to fuel production process

\subsection{Analytical Techniques}

GC-MS Analysis of HDPE, PP \& PS fuel instrument set up program is inspected properly such as Instrument Control Method, Channel Parameters, Auto sampler Method, Carriers Parameters and Valve Configuration and Settings. In Channel Parameter Data will be collected from Channel B, Delay Time: $0.00 \mathrm{~min}$, Run Time: $44.50 \mathrm{~min}$, Sampling Rate: $1.5625 \mathrm{pts} / \mathrm{s}$. Auto sampler Method is Syringe Capacity: 5.0 $\mu \mathrm{L}$, Injection Speed: Normal, Viscosity Delay: $0, \quad$ Pre-injection Solvent Washes:2,Post-injection Solvent Washes(A):6, Injection Volume:0.5 $\mu \mathrm{L}$, Sample Pumps : 6,Wash/Waste Vial Set:2, Pre-injection Sample Washes:2.Carrier Parameters, Carrier A control : Press-PSIG, Carrier A setpoint:0.0 PSIG. Carrier B control: P Flow-He, Column B length:30.00 m, Vacuum Compensation: ON, Split Flow:101.0 Ml/min,
Initial Set point: $1.00 \mathrm{ml} / \mathrm{min}$, Diameter: $250 \mu \mathrm{m}$ and Initial Hold:999.00 min. Valve Configuration Settings are Valve 1 : SPLIT On, Valve 2: SPLIT On, Valve 3:None, Valve 4:None,Valve 5: None, Valve 6: None. Detector Parameters, Detector A None, Range: 1, Time Constant 200, Autozero ON, Polarity: None. In Detector B Detector: None, Range: 1, Time Constant: 200, Autozero: ON, Polarity: None. Heated Zones, Injector A: CAP, Setpoint: OFF. Injector B: PSSI, Initial Set point: $280^{\circ} \mathrm{C}$, Initial Hold: $999.00 \mathrm{~min}$. Detector A: $0^{\circ} \mathrm{C}$, Detector B: $0^{\circ} \mathrm{C}$, Auxiliary (NONE): $0^{\circ} \mathrm{C}$. Oven Program Cryogenics: Off, Initial Temp: $40{ }^{\circ} \mathrm{C}$, Initial Hold: 1.00 min, Ramp 1:10.0 0/min to $325^{\circ} \mathrm{C}$, hold for $15.00 \mathrm{~min}$, Total Run Time 44.50 min, Maximum Temp: $330^{\circ} \mathrm{C}$, Equilibrium Time: $0.5 \mathrm{~min}$, Elite-5MS Perkin Elmer GC column used for fuel analysis and column length 30 meter, $0.25 \mathrm{mmID}, 0.5 \mathrm{u} \mathrm{m}$ df and maximum temperature $350^{\circ} \mathrm{C}$, cat \# N9316284. Compound 
identification used NIST library and fuel compound probability match with NIST library.

$\mathrm{GC}$ methods are sets as following criterion such as Instrument Identifier Version Number: 1.0, Duration (min): 45.0, Solvent Delay Start1: 0.0, Solvent Delay End1:1.0, Number of functions: 1 . Function 1: MS Scan, Time 1.00 to 44.50, Mass 35.00 to $528.00 \mathrm{EI}+$, Type: MS Scan, Ion Mode: EI+, Data Format: Centroid, Start Mass : 35.00, End Mass :528.00,Scan Time(Sec):0.25,Inter Scan Time (Sec):0.15,Start Time(min):1.00,End Time(min):44.50 as described.

FT-IR (Perkin Elmer) spectrum 100 was used for fuel analysis purposed. Total scan number 32 , resolution $4 \mathrm{~cm}^{-1}$ and scan range $4000-450 \mathrm{~cm}^{-1}$. NaCl cell was used for fuel holding inside the machine and cell thickness was 0.025 $\mathrm{mm}$. FTIR spectrum graph display was transmittance (\%T).

Perkin Elmer Differential Scanning calorimeter (DSC) was used for fuel analysis and carrier gas was used as a Nitrogen $\left(\mathrm{N}_{2}\right)$. Fuel analysis program temperature was used $5-400^{\circ} \mathrm{C}$ and temperature ramping rate $10{ }^{\circ} \mathrm{C} / \mathrm{min} .50 \mu \mathrm{L}$ aluminium pan was used for sample holding and carrier gas flow was $20 \mathrm{ml} / \mathrm{min}$.

\section{Results and Discussion}

\subsection{Pre Analysis Results}

Waste plastic raw materials trace metal analysis in water or aqueous matrices by ICP-AES, ASTM test method
ASTM D1976 is shown table 3. The trace metals are calculated and represented in unit of $\mathrm{mg} / \mathrm{L}$. We believe that the trace metals are originally present in the raw plastic samples and due the presence of these metals there is no further need for catalyst because most catalysts are derived from metal compounds. Plastics are manufactured by polymerization, polycondensation, or polyaddition reactions where monomeric molecules are joined sequentially under controlled conditions to produce high-molecular-weight polymers whose basic properties are defined by their composition, molecular weight distribution, and their degree of branching or cross-linking. To control the polymerization process, a broad range of structurally specific proprietary chemical compounds is used for polymerization initiation, breaking, and cross-linking reactions (peroxides, Ziegler-Natta, and metallocene catalysts). The polymerized materials are admixed with proprietary antioxidants (sterically hindered phenols, organophosphites),UVand light stability improvers (hindered amines and piperidyl esters), antistatic agents (ethoxylated amines), impact modifiers (methacrylatebutadiene- styrene compounds), heat stabilizers (methyl tin mercaptides), lubricants (esters), biostabilizers (arsine, thiazoline, and phenol compounds), and plasticizers used to modify the plasticity, softness, and pliability of plastics (phthalates and esters). World production of plastic additives is on the order of 18 billion pounds per year with plasticizers representing a $60 \%$ of the total amount[12].

Table 3. Waste Plastic to Trace Metal analysis by ICP

\begin{tabular}{|c|c|c|c|c|c|}
\hline $\begin{array}{l}\text { ASTM Test } \\
\text { Method }\end{array}$ & $\begin{array}{c}\text { Element } \\
\text { Name }\end{array}$ & Results, mg/L & $\begin{array}{c}\text { ASTM Test } \\
\text { Method }\end{array}$ & $\begin{array}{c}\text { Element } \\
\text { Name }\end{array}$ & Results, mg/L \\
\hline \multirow[t]{11}{*}{ ASTM D1976 } & Silver & $<1.0$ & ASTM D1976 & Molybdenum & $<1.0$ \\
\hline & Aluminium & $<1.0$ & & Sodium & 5,966 \\
\hline & Boron & $<1.0$ & & Nickel & $<1.0$ \\
\hline & Barium & $<1.0$ & & Phosphorus & $<1.0$ \\
\hline & Calcium & 30.5 & & Lead & $<1.0$ \\
\hline & Chromium & $<1.0$ & & Antimony & $<1.0$ \\
\hline & Copper & $<1.0$ & & Silicon & 5.3 \\
\hline & Iron & 3.9 & & Tin & $<1.0$ \\
\hline & Potassium & $<1.0$ & & Titanium & $<1.0$ \\
\hline & Lithium & $<1.0$ & & Vanadium & $<1.0$ \\
\hline & Magnesium & 2.8 & & Zinc & $<1.0$ \\
\hline
\end{tabular}

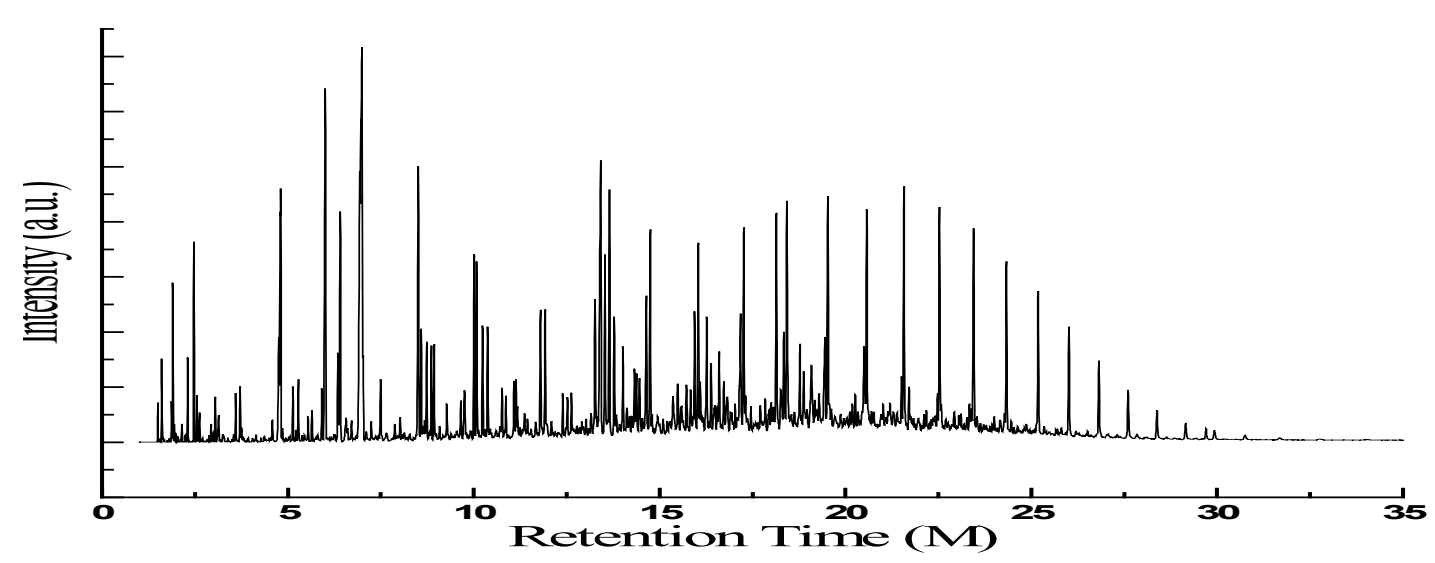

Figure 2. GC/MS chromatogram of HDPE, PP and PS waste plastics mixture to produce fuel 
Table 4. GC/MS chromatogram compound list of HDPE, PP and PS waste plastics mixture to produce fuel

\begin{tabular}{|c|c|c|c|c|c|c|}
\hline $\begin{array}{l}\text { Peak } \\
\text { Number }\end{array}$ & $\begin{array}{l}\text { Retention } \\
\text { Time } \\
(\mathrm{M}) \\
\end{array}$ & $\begin{array}{l}\text { Trace } \\
\text { Mass } \\
(\mathrm{m} / \mathrm{z}) \\
\end{array}$ & $\begin{array}{l}\text { Compound } \\
\text { Name }\end{array}$ & $\begin{array}{l}\text { Compound } \\
\text { Formula }\end{array}$ & $\begin{array}{l}\text { Molecular } \\
\text { Weight }\end{array}$ & $\begin{array}{l}\text { NIST } \\
\text { Number }\end{array}$ \\
\hline 1 & 1.50 & 39 & Cyclopropane & $\mathrm{C}_{3} \mathrm{H}_{6}$ & 42 & 18854 \\
\hline 2 & 1.60 & 39 & 1-Propene, 2-methyl- & $\mathrm{C}_{4} \mathrm{H}_{8}$ & 56 & 18910 \\
\hline 3 & 1.87 & 42 & Cyclopropane, ethyl- & $\mathrm{C}_{5} \mathrm{H}_{10}$ & 70 & 19072 \\
\hline 4 & 1.90 & 43 & Pentane & $\mathrm{C}_{5} \mathrm{H}_{12}$ & 72 & 291244 \\
\hline 5 & 2.30 & 43 & Pentane, 2-methyl- & $\mathrm{C}_{6} \mathrm{H}_{14}$ & 86 & 61279 \\
\hline 6 & 2.47 & 41 & 3-Hexene, (E)- & $\mathrm{C}_{6} \mathrm{H}_{12}$ & 84 & 114481 \\
\hline 7 & 2.56 & 41 & Hexane & $\mathrm{C}_{6} \mathrm{H}_{14}$ & 86 & 61280 \\
\hline 8 & 2.94 & 67 & 2,4-Hexadiene, (Z,Z)- & $\mathrm{C}_{6} \mathrm{H}_{10}$ & 82 & 113646 \\
\hline 9 & 3.04 & 56 & 1-Pentene, 2,4-dimethyl- & $\mathrm{C}_{7} \mathrm{H}_{14}$ & 98 & 114435 \\
\hline 10 & 3.14 & 81 & 2,4-Dimethyl 1,4-pentadiene & $\mathrm{C}_{7} \mathrm{H}_{12}$ & 96 & 114468 \\
\hline 11 & 3.59 & 41 & 1-Heptene & $\mathrm{C}_{7} \mathrm{H}_{14}$ & 98 & 107734 \\
\hline 12 & 3.71 & 41 & Heptane & $\mathrm{C}_{7} \mathrm{H}_{16}$ & 100 & 61276 \\
\hline 13 & 4.75 & 70 & Heptane, 4-methyl- & $\mathrm{C}_{8} \mathrm{H}_{18}$ & 114 & 113916 \\
\hline 14 & 4.81 & 92 & 1,5-Heptadien-3-yne & $\mathrm{C}_{7} \mathrm{H}_{8}$ & 92 & 759 \\
\hline 15 & 5.13 & 41 & 1-Octene & $\mathrm{C}_{8} \mathrm{H}_{16}$ & 112 & 1604 \\
\hline 16 & 5.28 & 43 & Octane & $\mathrm{C}_{8} \mathrm{H}_{18}$ & 114 & 229407 \\
\hline 17 & 5.54 & 69 & Cyclopentane, 1,1,3,4-tetramethyl-, cis- & $\mathrm{C}_{9} \mathrm{H}_{18}$ & 126 & 27589 \\
\hline 18 & 5.65 & 43 & Hexane, 3-ethyl- & $\mathrm{C}_{8} \mathrm{H}_{18}$ & 114 & 113940 \\
\hline 19 & 5.91 & 69 & Cyclohexane, 1,3,5-trimethyl-, $(1 \alpha, 3 \alpha, 5 \alpha)$ - & $\mathrm{C}_{9} \mathrm{H}_{18}$ & 126 & 2479 \\
\hline 20 & 6.01 & 42 & 2,4-Dimethyl-1-heptene & $\mathrm{C}_{9} \mathrm{H}_{18}$ & 126 & 113516 \\
\hline 21 & 6.35 & 69 & Cyclohexane, 1,3,5-trimethyl-, $(1 \alpha, 3 \alpha, 5 \beta)$ - & $\mathrm{C}_{9} \mathrm{H}_{18}$ & 126 & 2480 \\
\hline 22 & 6.41 & 91 & Ethylbenzene & $\mathrm{C}_{8} \mathrm{H}_{10}$ & 106 & 158804 \\
\hline 23 & 6.93 & 104 & Bicyclo[4.2.0]octa-1,3,5-triene & $\mathrm{C}_{8} \mathrm{H}_{8}$ & 104 & 34631 \\
\hline 24 & 6.99 & 105 & Benzene, 1-azido-4-methyl- & $\mathrm{C}_{7} \mathrm{H}_{7} \mathrm{~N}_{3}$ & 133 & 39082 \\
\hline 25 & 7.24 & 55 & 3-Octyne, 2-methyl- & $\mathrm{C}_{9} \mathrm{H}_{16}$ & 124 & 62452 \\
\hline 26 & 7.50 & 105 & Benzene, (1-methylethyl)- & $\mathrm{C}_{9} \mathrm{H}_{12}$ & 120 & 228742 \\
\hline 27 & 7.88 & 117 & Benzene, cyclopropyl- & $\mathrm{C}_{9} \mathrm{H}_{10}$ & 118 & 113961 \\
\hline 28 & 8.02 & 91 & Benzene, propyl- & $\mathrm{C}_{9} \mathrm{H}_{12}$ & 120 & 113930 \\
\hline 29 & 8.51 & 117 & Benzene, cyclopropyl- & $\mathrm{C}_{9} \mathrm{H}_{10}$ & 118 & 113961 \\
\hline 30 & 8.59 & 41 & 1-Decene & $\mathrm{C}_{10} \mathrm{H}_{20}$ & 140 & 118883 \\
\hline 31 & 8.73 & 43 & Decane & $\mathrm{C}_{10} \mathrm{H}_{22}$ & 142 & 114147 \\
\hline 32 & 8.86 & 43 & Octane, 3,5-dimethyl- & $\mathrm{C}_{10} \mathrm{H}_{22}$ & 142 & 114062 \\
\hline 33 & 8.93 & 43 & Decane, 4-methyl- & $\mathrm{C}_{11} \mathrm{H}_{24}$ & 156 & 113875 \\
\hline 34 & 9.27 & 117 & Benzene, 2-propenyl- & $\mathrm{C}_{9} \mathrm{H}_{10}$ & 118 & 114744 \\
\hline 35 & 9.65 & 43 & 1-Nonene, 4,6,8-trimethyl- & $\mathrm{C}_{12} \mathrm{H}_{24}$ & 168 & 6413 \\
\hline 36 & 9.75 & 91 & $1,2,3,4,5,8$-Hexahydronaphthalene & $\mathrm{C}_{10} \mathrm{H}_{14}$ & 134 & 113559 \\
\hline 37 & 10.01 & 69 & Cyclooctane, 1,4-dimethyl-, cis- & $\mathrm{C}_{10} \mathrm{H}_{20}$ & 140 & 61409 \\
\hline 38 & 10.08 & 55 & Cyclooctane, 1,4-dimethyl-, cis- & $\mathrm{C}_{10} \mathrm{H}_{20}$ & 140 & 61409 \\
\hline 39 & 10.24 & 41 & 1-Decene & $\mathrm{C}_{10} \mathrm{H}_{20}$ & 140 & 118883 \\
\hline 40 & 10.38 & 43 & Undecane & $\mathrm{C}_{11} \mathrm{H}_{24}$ & 156 & 114185 \\
\hline 41 & 10.76 & 41 & $\begin{array}{c}\text { Ethanone, 1-(1,2,2,3-tetramethylcyclopentyl)-, } \\
\text { (1R-cis)- }\end{array}$ & $\mathrm{C}_{11} \mathrm{H}_{20} \mathrm{O}$ & 168 & 186082 \\
\hline 42 & 10.87 & 41 & 2-Undecanethiol, 2-methyl- & $\mathrm{C}_{12} \mathrm{H}_{26} \mathrm{~S}$ & 202 & 9094 \\
\hline 43 & 11.08 & 91 & Benzene, (3-methyl-3-butenyl)- & $\mathrm{C}_{11} \mathrm{H}_{14}$ & 146 & 113578 \\
\hline 44 & 11.14 & 69 & 1,12-Tridecadiene & $\mathrm{C}_{13} \mathrm{H}_{24}$ & 180 & 7380 \\
\hline 45 & 11.37 & 91 & Benzene, pentyl- & $\mathrm{C}_{11} \mathrm{H}_{16}$ & 148 & 113915 \\
\hline 46 & 11.80 & 41 & 3-Dodecene, (E)- & $\mathrm{C}_{12} \mathrm{H}_{24}$ & 168 & 113960 \\
\hline 47 & 11.92 & 43 & Dodecane & $\mathrm{C}_{12} \mathrm{H}_{26}$ & 170 & 291499 \\
\hline 48 & 12.39 & 43 & Decane, 2,3,5,8-tetramethyl- & $\mathrm{C}_{14} \mathrm{H}_{30}$ & 198 & 149589 \\
\hline 49 & 13.27 & 41 & 7-Tetradecene & $\mathrm{C}_{14} \mathrm{H}_{28}$ & 196 & 70643 \\
\hline 50 & 13.41 & 69 & 1-Decanol, 2-methyl- & $\mathrm{C}_{11} \mathrm{H}_{24} \mathrm{O}$ & 172 & 185011 \\
\hline 51 & 13.53 & 43 & 1-Nonene, 4,6,8-trimethyl- & $\mathrm{C}_{12} \mathrm{H}_{24}$ & 168 & 6413 \\
\hline
\end{tabular}




\begin{tabular}{|c|c|c|c|c|c|c|}
\hline 52 & 13.65 & 43 & 1-Octanol, 2-butyl- & $\mathrm{C}_{12} \mathrm{H}_{26} \mathrm{O}$ & 186 & 114639 \\
\hline 53 & 13.78 & 91 & Benzene, heptyl- & $\mathrm{C}_{13} \mathrm{H}_{20}$ & 176 & 118464 \\
\hline 54 & 14.01 & 43 & Trichloroacetic acid, hexadecyl ester & $\mathrm{C}_{18} \mathrm{H}_{33} \mathrm{Cl}_{3} \mathrm{O}_{2}$ & 386 & 280518 \\
\hline 55 & 14.32 & 43 & 2-Undecanethiol, 2-methyl- & $\mathrm{C}_{12} \mathrm{H}_{26} \mathrm{~S}$ & 202 & 9094 \\
\hline 56 & 14.64 & 41 & 1-Tetradecene & $\mathrm{C}_{14} \mathrm{H}_{28}$ & 196 & 34720 \\
\hline 57 & 14.75 & 43 & Tetradecane & $\mathrm{C}_{14} \mathrm{H}_{30}$ & 198 & 113925 \\
\hline 58 & 15.36 & 43 & Tetradecane, 2,6,10-trimethyl- & $\mathrm{C}_{17} \mathrm{H}_{36}$ & 240 & 11556 \\
\hline 59 & 15.94 & 41 & 1-Pentadecene & $\mathrm{C}_{15} \mathrm{H}_{30}$ & 210 & 69726 \\
\hline 60 & 16.05 & 43 & Pentadecane & $\mathrm{C}_{15} \mathrm{H}_{32}$ & 212 & 107761 \\
\hline 61 & 17.17 & 41 & 1-Hexadecene & $\mathrm{C}_{16} \mathrm{H}_{32}$ & 224 & 118882 \\
\hline 62 & 17.27 & 71 & Hexadecane & $\mathrm{C}_{16} \mathrm{H}_{34}$ & 226 & 114191 \\
\hline 63 & 17.84 & 91 & Benzene,[3-(2-cyclohexylethyl)-6-cyclopentylhexyl]- & $\mathrm{C}_{25} \mathrm{H}_{40}$ & 340 & 23414 \\
\hline 64 & 18.14 & 105 & Benzene, 1,1'-(1,3-propanediyl)bis- & $\mathrm{C}_{15} \mathrm{H}_{16}$ & 196 & 133399 \\
\hline 65 & 18.42 & 57 & Heptadecane & $\mathrm{C}_{17} \mathrm{H}_{36}$ & 240 & 107308 \\
\hline 66 & 18.88 & 91 & Benzeneacetic acid, 4-tetradecyl ester & $\mathrm{C}_{22} \mathrm{H}_{36} \mathrm{O}_{2}$ & 332 & 282026 \\
\hline 67 & 19.45 & 41 & 1-Nonadecene & $\mathrm{C}_{19} \mathrm{H}_{38}$ & 266 & 113626 \\
\hline 68 & 19.52 & 71 & Octadecane & $\mathrm{C}_{18} \mathrm{H}_{38}$ & 254 & 57273 \\
\hline 69 & 20.50 & 43 & 1-Hexadecanol, 2-methyl- & $\mathrm{C}_{17} \mathrm{H}_{36} \mathrm{O}$ & 256 & 36540 \\
\hline 70 & 20.57 & 43 & Eicosane & $\mathrm{C}_{20} \mathrm{H}_{42}$ & 282 & 290513 \\
\hline 71 & 21.51 & 41 & 1-Eicosene & $\mathrm{C}_{20} \mathrm{H}_{40}$ & 280 & 13488 \\
\hline 72 & 21.57 & 56 & Eicosane & $\mathrm{C}_{20} \mathrm{H}_{42}$ & 282 & 290513 \\
\hline 73 & 22.48 & 43 & 1-Docosene & $\mathrm{C}_{22} \mathrm{H}_{44}$ & 308 & 113878 \\
\hline 74 & 22.53 & 85 & Heneicosane & $\mathrm{C}_{21} \mathrm{H}_{44}$ & 296 & 107569 \\
\hline 75 & 23.45 & 57 & Heneicosane & $\mathrm{C}_{21} \mathrm{H}_{44}$ & 296 & 107569 \\
\hline 76 & 25.19 & 43 & Octacosane & $\mathrm{C}_{28} \mathrm{H}_{58}$ & 394 & 134306 \\
\hline 77 & 27.60 & 57 & Octacosane & $\mathrm{C}_{28} \mathrm{H}_{58}$ & 394 & 134306 \\
\hline 78 & 28.38 & 57 & Heptacosane & $\mathrm{C}_{27} \mathrm{H}_{56}$ & 380 & 79427 \\
\hline 79 & 29.15 & 57 & Heptacosane & $\mathrm{C}_{27} \mathrm{H}_{56}$ & 380 & 79427 \\
\hline 80 & 29.70 & 306 & 1,1':3',1"-Terphenyl, 5'-phenyl- & $\mathrm{C}_{24} \mathrm{H}_{18}$ & 306 & 57402 \\
\hline 81 & 29.93 & 57 & Heptacosane & $\mathrm{C}_{27} \mathrm{H}_{56}$ & 380 & 79427 \\
\hline
\end{tabular}

\subsection{Characterization of the Liquids}

In accordance with trace mass and retention time a lots of different types of compound appear in the HDPE, PP\& PS fuel showed figure 2 and table 4 . In the initial preface of the analysis retention time 1.50 , trace mass 39 compound is Cyclopropane $\left(\mathrm{C}_{3} \mathrm{H}_{6}\right)$, retention time at 1.60 , trace mass 39 , compound is 1-Propene-2-Methyl $\left(\mathrm{C}_{4} \mathrm{H}_{8}\right)$, retention time 1.87 , trace mass 42 compound is Cyclo-propane ethyl$\left(\mathrm{C}_{5} \mathrm{H}_{10}\right)$, retention time 1.90 , trace mass $43,\left(\mathrm{C}_{5} \mathrm{H}_{12}\right)$,comp ound is Pentane, retention time 2.30, trace Mass 43 ,compound is Pentane-2-Methyl- $\left(\mathrm{C}_{6} \mathrm{H}_{14}\right)$,retention time 2.47, trace mass 41,3 -Hexene, $(\mathrm{E})-\left(\mathrm{C}_{6} \mathrm{H}_{12}\right)$, retention time 2.56, trace mass 41 , compound is Hexane $\left(\mathrm{C}_{6} \mathrm{H}_{14}\right)$, retention time 2.94, trace mass 67 , compound is 2,4 -Hexadiene, $(\mathrm{Z}, \mathrm{Z})-\left(\mathrm{C}_{6} \mathrm{H}_{10}\right)$, retention time 3.04, trace mass 56, compound is 1-Pentene,2,4-dimethyl- $\left(\mathrm{C}_{7} \mathrm{H}_{14}\right)$, retention time 3.14,trace mass 81, compound is 2,4-Dimethyl 1,4-pentadiene $\left(\mathrm{C}_{7} \mathrm{H}_{12}\right)$, retention time 3.59, trace mass 41 , compound name is 1 -Heptene $\left(\mathrm{C}_{7} \mathrm{H}_{14}\right)$, retention time 3.71 ,trace mass 41 , compound is Heptane, retention time 4.75, trace mass 70, compound is Heptane, 4-methyl$\left(\mathrm{C}_{8} \mathrm{H}_{18}\right)$, retention time 4.81 , trace mass 92 , compound is 1,5-Heptadiene-3-yne, $\left(\mathrm{C}_{7} \mathrm{H}_{8}\right)$, retention time 5.13, trace mass 41 , compound is 1 -Octene, retention time 5.28 , trace mass 43, compound is Octane, retention time5.54, trace mass 69, compound is Cyclopentane,1,1,3,4-tetramethyl-,ci $\mathrm{s}-\left(\mathrm{C}_{9} \mathrm{H}_{18}\right)$, retention time 5.65 , trace mass 43 , compound is Hexane,3-ethyl- $\left(\mathrm{C}_{8} \mathrm{H}_{18}\right)$, retention time 6.01 , trace mass 42 , compound is 2,4-Dimethyl-1-heptene $\left(\mathrm{C}_{9} \mathrm{H}_{18}\right)$, retention time 6.35, trace mass 69, compound is Cyclohexane, 1,3,5-trimethyl-, $(1 \alpha, 3 \alpha, 5 \alpha)-\left(\mathrm{C}_{9} \mathrm{H}_{18}\right)$, retention time 6.35 , trace mass 69, compound is Cyclohexane, 1,3,5-trimethyl-, $(1 \alpha, 3 \alpha, 5 \beta)-\left(\mathrm{C}_{9} \mathrm{H}_{18}\right)$.In low retention time and low trace mass small structure compounds are derived and high retention time high and high trace mass large and bulky structure compounds are appeared. In the middle of analysis high retention time and high trace mass are seen such as retention time 7.50, trace mass 105, compound isBenzene,1-azido-4-methyl)-( $\mathrm{C}_{9} \mathrm{H}_{1}$ 2 ), retention time 7.88 , trace mass 117 ,compound is Benzene, cyclopropyl- $\left(\mathrm{C}_{9} \mathrm{H}_{10}\right)$, retention time 8.02, trace mass 91, compound is Benzene, propyl- $\left(\mathrm{C}_{9} \mathrm{H}_{12}\right)$, retention time 8.51, trace mass 117, compound is Benzene,Cycloprop yl-, $\left(\mathrm{C}_{9} \mathrm{H}_{10}\right)$, benzene derivatives compound are available because in the raw structure of the PS-6 styrene groups are appeared. Retention time 8.59 , trace mass 41 , compound is 1-Decene $\left(\mathrm{C}_{10} \mathrm{H}_{20}\right)$, retention time 9.75 , trace mass 91 , compound is $1,2,3,4,5,8$-Hexahydronapthalene $\left(\mathrm{C}_{10} \mathrm{H}_{14}\right)$, retention time 10.01, trace mass 69 , compound is 
Cyclooctane,1,4-dimethyl-,cis- $\left(\mathrm{C}_{10} \mathrm{H}_{20}\right)$ and retention time 10.38, trace mass 43 , compound is Undecane $\left(\mathrm{C}_{11} \mathrm{H}_{24}\right)$.Eventually we noticed in high retention time and low trace mass some higher carbon number compound are appeared such as retention time 12.39, trace mass 43, compound is Decane,2,3,5,8-tetramethyl $\left(\mathrm{C}_{14} \mathrm{H}_{30}\right)$, retention time 15.94, trace mass 41, compound is 1-Pentadecene $\left(\mathrm{C}_{15} \mathrm{H}_{30}\right)$, retention time 19.45 , trace mass, trace mass 41,compound is 1-Nonadecene $\left(\mathrm{C}_{19} \mathrm{H}_{38}\right)$, retention time 20.57, trace mass 43.compound is Eicosane $\left(\mathrm{C}_{20} \mathrm{H}_{42}\right)$, retention time 21.51 , trace mass 41 compound is 1-Eicosane $\left(\mathrm{C}_{10} \mathrm{H}_{40}\right)$, retention time 25.19 , trace mass 43 , compound is Octacosane $\left(\mathrm{C}_{28} \mathrm{H}_{58}\right)$ etc. On the other hand in some field high retention time and high trace mass also larger hydrocarbon compound are noticed such as retention time 22.53, trace mass 85 , compound is Heneicosane $\left(\mathrm{C}_{21} \mathrm{H}_{44}\right)$, retention time 28.38 , trace mass 57 , compound is Heptacosane $\left(\mathrm{C}_{27} \mathrm{H}_{56}\right)$ and retention time 29.70, trace mass 306 , compound is $1,1^{\prime}: 3^{\prime}, 1{ }^{\prime}$-Tetraphenyl, 5'-Phenyl- $\left(\mathrm{C}_{24} \mathrm{H}_{18}\right)$ respectively.

FT-IR analysis of HDPE (High Density Polyethylene, Structure is $-\mathrm{CH}_{2}-\mathrm{CH}_{2}$ ), PP (Polypropylene, Structure is $-\mathrm{CH}_{2}-\mathrm{C}-\mathrm{CH}_{3}-\mathrm{CH}-$ ) and PS (Polystyrene, Structure is $\mathrm{C}_{6} \mathrm{H}_{5}-\mathrm{CH}_{3}-\mathrm{CH}_{2}-$ ) produced fuel (figure 3) following types of wave number and functional group are appeared in the spectrum. In the experiment if we noticed to the raw structure of three individual polymer such as in the HDPE ,C-C and C-H bonds appeared that's double bonds breaking down to single bonds after the polymerization of polyethylene. In PP and PS methyl group and styrene groups are appeared with double and single bond of Carbon to Carbon. As a result after completed the three mixed polymer reaction different types of products are produced with different characteristics. In the analysis many wave number and functional groups are found, as example wave number $2920.75\left(\mathrm{~cm}^{-1}\right)$ and $2729.44\left(\mathrm{~cm}^{-1}\right)$, functional group is $\mathrm{C}^{-\mathrm{CH}_{3}}$, wave number $1871.43\left(\mathrm{~cm}^{-1}\right), 1816.42$ $\left(\mathrm{cm}^{-1}\right), 1798.12\left(\mathrm{~cm}^{-1}\right), 1743.42\left(\mathrm{~cm}^{-1}\right)$ and $1720.61\left(\mathrm{~cm}^{-1}\right)$ functional group is Non-Conjugated, wave number 1649.84 $\left(\mathrm{cm}^{-1}\right)$ and $1603.61\left(\mathrm{~cm}^{-1}\right)$ functional group is Conjugated, wave number $1442.98\left(\mathrm{~cm}^{-1}\right)$ and $1377.99\left(\mathrm{~cm}^{-1}\right)$ functional group is $\mathrm{CH}_{3}$,wave number $1029.80\left(\mathrm{~cm}^{-1}\right)$ and 1020.57 $\left(\mathrm{cm}^{-1}\right)$ functional group is Acetates and ultimately wave number $989.94\left(\mathrm{~cm}^{-1}\right)$ and $910.97\left(\mathrm{~cm}^{-1}\right)$ functional group is $-\mathrm{CH}=\mathrm{CH}_{2}$ as well. Different types of functional groups are found from the analysis such as some functional groups are bulky $\left(\mathrm{C}_{-}-\mathrm{CH}_{3}\right)$ and some functional groups are compact $\left(\mathrm{CH}_{3}\right)$. The bands at 2920.75 and $2729.44\left(\mathrm{~cm}^{-1}\right)$ strong $\mathrm{C}-\mathrm{H}$ stretches indicate methyl group is present with single carbon bond that's alkane. The band at 1442.98 and $1377.99\left(\mathrm{~cm}^{-1}\right), \mathrm{CH}_{3}$ is umbrella mode and band at 989.94 and $910.97\left(\mathrm{~cm}^{-1}\right),-\mathrm{CH}=\mathrm{CH}_{2}, \mathrm{C}=\mathrm{C}$ asymmetric stretching that is out of plane $\mathrm{C}-\mathrm{H}$ bend. Energy values are calculated, using formula is $\mathrm{E}=\mathrm{h} v$, Where $\mathrm{h}=\mathrm{Planks}$ Constant, $\mathrm{h}$ $=6.626 \times 10^{-34} \mathrm{~J}, v=$ Frequency in Hertz $\left(\mathrm{sec}^{-1}\right)$, Where $v=\mathrm{c} / \lambda$, $\mathrm{c}=$ Speed of light, where, $\mathrm{c}=3 \times 10^{10} \mathrm{~m} / \mathrm{s}, \mathrm{W}=1 / \lambda$, where $\lambda$ is wave length and $\mathrm{W}$ is wave number in $\mathrm{cm}^{-1}$. Therefore the equation $\mathrm{E}=\mathrm{h} v$, can substitute by the following equation, $\mathrm{E}=\mathrm{hcW}$. According to their wave number such as for $2920.75\left(\mathrm{~cm}^{-1}\right)$ calculated energy, $E=5.80 \times 10^{-20} \mathrm{~J}$. Similarly, wave number $1871.43\left(\mathrm{~cm}^{-1}\right)$ energy, $\mathrm{E}=3.61 \times 10^{-20} \mathrm{~J}$, wave number $1377.99\left(\mathrm{~cm}^{-1}\right)$ energy, $E=2.73 \times 10^{-20} \mathrm{~J}$ and eventually wave number $989.94\left(\mathrm{~cm}^{-1}\right)$ functional group is $1.97 \times 10^{-20} \mathrm{~J}$ respectively. From this analysis we can conclude functional group band energy is can convert into calorific value for fuel calorific energy.

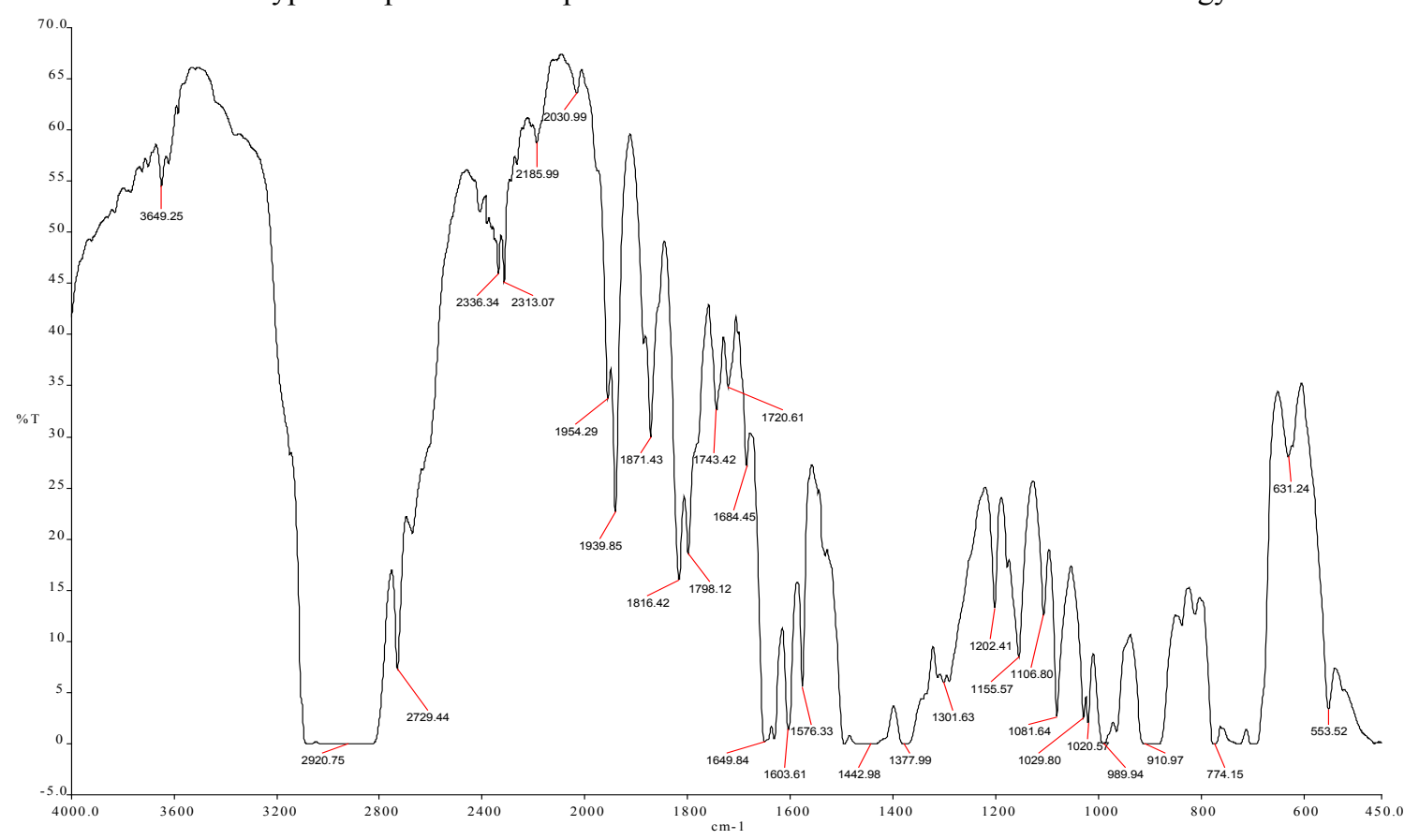

Figure 3. FT-IR spectrum of HDPE, PP and PS waste plastics mixture to produce fuel 


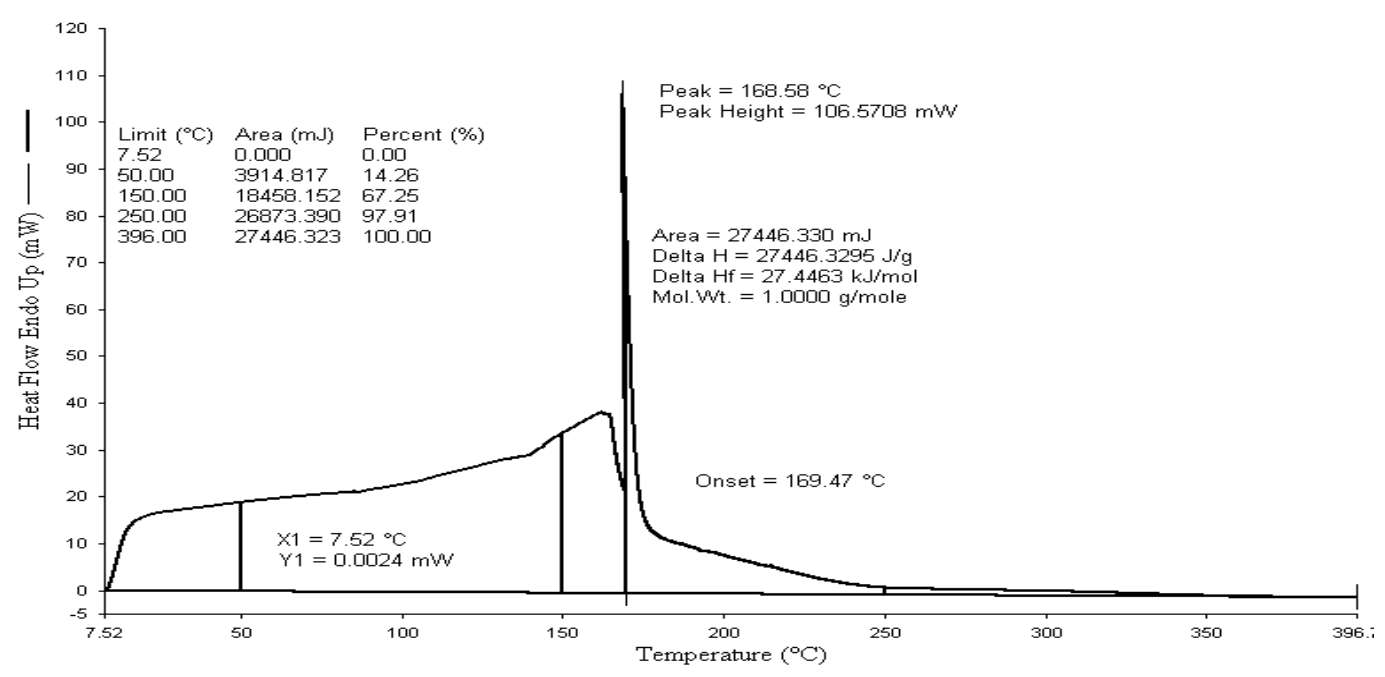

Figure 4. DSC graph of HDPE, PP and PS waste plastics mixture to produce fuel

Perkin Elmer Differential Scanning Calorimeter (DSC) analysis of produced fuel from HDPE/PP/PS waste plastic is shown figure 4. DSC equipment use for boiling point and enthalpy value measurement for produced fuel. $50 \mu \mathrm{L}$ fuels is use for boiling point and enthalpy value measurement. Program temperature was used $5-400^{\circ} \mathrm{C}$ and temperature ramping rate $20^{\circ} \mathrm{C} / \mathrm{min}$. Total experiment run time 19.75 minutes. From DSC fuel analysis graph shown onset temperature $169.47^{\circ} \mathrm{C}$ and peak temperature is shown $168.58{ }^{\circ} \mathrm{C}$. Fuel boiling point indicating $168.58^{\circ} \mathrm{C}$ which is need heat flow Endo up $106.5708 \mathrm{~mW}$. Fuel graph peak area $27446.330 \mathrm{~mJ}$, delta enthalpy value $\mathrm{H}$ is 27446.3295 $\mathrm{J} / \mathrm{g}$ and Mol. Wt.1.0000 g/mole. Produce fuel has small structure hydrocarbon compounds and longer hydrocarbon compound present in this fuel. When heat start for boiling this fuel by using DSC we noticed that fuel boil start at low temperature below room temperature because this fuel has also low boiling point compound also such as Cyclopropane compound. DSC analysis result showed boiling peak intensity high of this fuel, because of this fuel has some aromatic related hydrocarbon compounds.

\section{Conclusions}

The mixture of HDPE, PP, and PS waste plastic was converted to liquid hydrocarbon fuel utilizing thermal degradation process. Analysis showed that liquid product contained aromatic and aliphatic carbon compounds. The thermal degradation was carried out in a stainless steel reactor and results show that this method is effective and efficient way to converted vast amounts of waste plastic to useful source of energy. GC/MS analysis result shown there types mixture of waste plastic to produce liquid hydrocarbon fuel has short range hydrocarbon chain $\mathrm{C}_{3}$ to longer chain hydrocarbon $\mathrm{C}_{28}$ with high olefin content. Produced fuel has aromatic related hydrocarbon such as Ethylbenzene, Benzene, 1-azido-4-methyl-, Benzene, cyclopropyl-, Benzene, 2-propenyl-, Benzene, (3-methyl-3butenyl)-, Benzene, heptyl-, Benzene, 1,1'-(1,3-propanediyl) and we believe these compounds came from the PS plastics because PS have benzene group present in its chemical structure. It is demonstrated that the conversion of post-consumer polymer waste PP/HDPE/PS to liquid hydrocarbon fuel was $90 \%$ and light gas production was less than $5 \%$. The experimental temperature of 25 to $430^{\circ} \mathrm{C}$ produced a suitable liquid hydrocarbon fuel with good efficiency.

\section{ACKNOWLEDGEMENTS}

The author acknowledges the support of Dr. Karin Kaufman, the founder of Natural State Research, Inc. The authors also acknowledge the valuable contributions NSR laboratory team members during the preparation of this manuscript.

\section{REFERENCES}

[1] Achilias DS, Roupakias C, Magalokonomosa P, Lappas AA, Antonakou EV. Chemical recycling of plastic wastes made from polyethylene (LDPE and HDPE) and polypropylene (PP), Journal of Hazardous Materials 2007; 149:536-542.

[2] Muthaa NH, Patel M, Premnath V. Plastics materials flow analysis for India. Resources. Conservation and Recycling 2006; 47:222-244.

[3] Buekens AG, Huang H. Catalytic plastics cracking for recovery of gasoline range hydrocarbons from municipal plastic wastes. Resources Conservation and Recycling 1998; 23:163-181.

[4] Misklczia N, Barthaa L, Deak G, Jover B. Thermal degradation of municipal plastic waste for the production of fuel like hydrocarbons. Polymer Degradation and Stability 2004; 86:357-366.

[5] Balakrishnan RK, Guria C. Thermal degradation of polystyrene in the presence of hydrogen by catalysts in solution. Polymer Degradation and Stability 2007; 
92:1583-1591.

[6] Luo G, Suto T, Yasu S, Kato K. Catalytic degradation of high density polyethylene and polypropylene into liquid fuel in a powder-particle fluidized bed. Polymer Degradation and Stability 2007; 70: 97-102.

[7] Kim H, Lee JW. Effect of ultrasonic wave on the degradation of polypropylene melt and morphology of its blend with polystyrene. Polymer 2002; 43:2585-2590.

[8] Yanga J, Mirand R, Roy C. Using the DTG curve fitting method to determine the apparent kinetic parameters of thermal decomposition of polymers. Polym Degrad Stab 2001; 73:455-461.
[9] Lin YH, Sharratt PN. Conversion of waste plastics to hydrocarbons by zeolited catalytic pyrolysis. J Chinese Inst Environ Eng 2000; 10:271-277.

[10] Chirico AD, Armanini M, Chini P, Cioccolo P, Provasoli F, Audisio G. Flame retardants for polypropylene based on lignin. Polym Degrad Stab 2002; 79:139-145

[11] Jayaraman K. Manufacturing sisal-polypropylene composites with minimum fibre degradation. Compos Sci Technol 2003; 63:367-374.

[12] Tullo, A. H. Plastic additives. Chem. Eng. News 2003, 81 (46), 36-42. 